\title{
Homicide and Legal Intervention
}

National Cancer Institute

\section{Source}

National Cancer Institute. Homicide and Legal Intervention. NCI Thesaurus. Code C105735.

Causes of death attributable to homicide or resulting from legal police intervention. 